
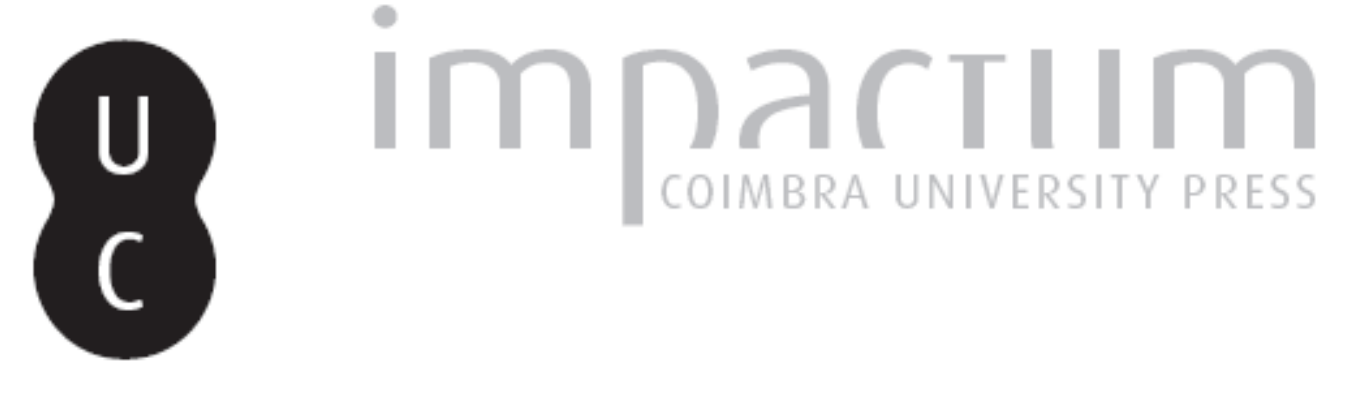

La utilización de los espacios verdes: estudio de caso en tres ciudades españolas

Autor(es): $\quad$ Gómez Gonçalves, Alejandro

Publicado por: $\begin{aligned} & \text { Faculdade de Letras da Universidade de Coimbra, Departamento de } \\ & \text { Geografia }\end{aligned}$

URL

persistente:

URI:http://hdl.handle.net/10316.2/30220

DOI:

DOI:http://dx.doi.org/10.14195/0871-1623_31_17

Accessed : $\quad$ 26-Apr-2023 16:15:30

A navegação consulta e descarregamento dos títulos inseridos nas Bibliotecas Digitais UC Digitalis, UC Pombalina e UC Impactum, pressupõem a aceitação plena e sem reservas dos Termos e Condições de Uso destas Bibliotecas Digitais, disponíveis em https://digitalis.uc.pt/pt-pt/termos.

Conforme exposto nos referidos Termos e Condições de Uso, o descarregamento de títulos de acesso restrito requer uma licença válida de autorização devendo o utilizador aceder ao(s) documento(s) a partir de um endereço de IP da instituição detentora da supramencionada licença.

Ao utilizador é apenas permitido o descarregamento para uso pessoal, pelo que o emprego do(s) título(s) descarregado(s) para outro fim, designadamente comercial, carece de autorização do respetivo autor ou editor da obra.

Na medida em que todas as obras da UC Digitalis se encontram protegidas pelo Código do Direito de Autor e Direitos Conexos e demais legislação aplicável, toda a cópia, parcial ou total, deste documento, nos casos em que é legalmente admitida, deverá conter ou fazer-se acompanhar por este aviso.

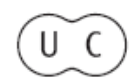




\section{La utilización de los espacios verdes. Estudio de caso en tres ciudades españolas.}

\section{Alejandro Gómez Gonçalves}

Departamento de Geografía. Facultad de Geografía e Historia. Universidad de Salamanca. algomez@usal.es

\section{Resumo:}

A Utilização dos espaços verdes. Estudo de caso em três cidades espanholas.

Num mundo cada vez mais urbanizado, os espaços verdes são, por vezes, o único contacto dos cidadãos com a natureza. Estes espaços proporcionam uma série de benefícios, tanto para os habitantes como para o meio ambiente da cidade, oferecendo a possibilidade de realizar neles uma serie de actividades de ócio e de convívio na proximidade da residência. No entanto, quando as autoridades têm de planificar o espaço verde urbano utilizam, por vezes, padrões subjetivos, muito influenciados pelos valores estéticos e pelas modas duma determinada época. 0 objectivo desta comunicação é conhecer as pessoas que visitam os espaços verdes de três cidades espanholas: Salamanca, Valladolid e Zamora, situadas na Comunidade Autônoma de Castela e Leão.

Os utilizadores destes espaços públicos foram estratificados tendo em conta a idade, género, ocupação, freqüência e o horário das visitas. Foram, ainda, analisadas as atividades aí realizadas e foram comparadas entre os grandes grupos de idade.

Palavras-chave: Espaços verdes. Cidades. Salamanca. Valladolid. Zamora.

\section{Résumé:}

L'utilisation des espaces verts. Étude de cas dans trois villes espagnoles.

Dans un monde de plus en plus urbanisé, les espaces verts urbaines sont, parfois, le unique contact des citoyens avec la nature. Ces espaces fournissent une série de bénéficies tant pour les personnes comme pour le moyenne ambiance urbaine, offrant la possibilité d'organiser une série d'activités de loisir et de convivialité près du lieu de résidence. Toutefois, lorsque les autorités doivent planifier les espaces verts elles parfois utilisent normes subjectives, très influencés pour les valeurs esthétiques et pour la mode d'une déterminé époque. L'objectif de cette communication est connaitre les personnes que visitent les espaces verts dans trois villes espagnoles: Salamanca, Valladolid et Zamora, situés dans la région de Castilla et Leon.

Les utilisateurs de ces espaces publics ont été stratifiés en fonction de l'âge, le sexe, la profession, la fréquence et les horaires de visites. Nous avons également examiné les activités qu'ils ont fait et on été comparés entre les groupes selon l'âge.

Mots-clés: Espaces verts. Villes. Salamanca. Valladolid. Zamora.

\section{Abstract:}

The use of green spaces. Case study in three Spanish cities.

In a world increasingly more urbanized green spaces are sometimes for citizens, their only contact with nature. These urban areas provide some benefits for the inhabitants and to the environment of the city, offering the possibility to practice some activities of leisure and coexistence close to their residences. However, when the authorities have to design green spaces, they sometimes use patterns strongly influenced by subjective aesthetic values and by the fashion of particular time. The aim of this paper is identify people who visit green urban spaces of three Spanish cities: Salamanca, Valladolid and Zamora, located in the region of Castilla and Leon.

The users of these spaces were stratified considering age, gender, occupation, frequency and hour of visits. It also analyzed and compared their activities between the large age groups.

Key-words: Green spaces. Cities. Salamanca. Valladolid. Zamora. 


\section{Introducción}

Según las estimaciones realizadas por las $\mathrm{Na}$ ciones Unidas en el año 2008 la población urbana habría superado a la rural por primera vez en la historia humana (UNFPA, 2007). En un mundo de ciudades los espacios verdes se ha convertido, con frecuencia, en el único contacto de las personas con la naturaleza. Aunque hasta fechas muy recientes el verde urbano fue visto únicamente como un elemento decorativo, son muchas las investigaciones que han demostrado los beneficios que reporta tanto a la sociedad como al medio ambiente de la ciudad. Las funciones desempeñadas por estos espacios se pueden agrupar en cinco grandes conjuntos: ecológicas, económicas, sociales, de planificación urbana y multidisciplinares (BAYCAN-LEVENT et al., 2004). En la actualidad, la presencia de las áreas verdes en las ciudades se ha convertido en un derecho de los ciudadanos (SAnesi y Chiarello, 2006), siendo las administraciones públicas las encargadas de su construcción y de su mantenimiento.

Desde que se celebró la Conferencia de las Naciones Unidas en Río de Janeiro en el año 1992, comenzaron a elaborarse indicadores sobre el estado de los ecosistemas urbanos que incluiría las zonas verdes de la ciudad. Sin embargo, existe muy poca información sobre quienes son los usuarios de los mismos y qué actividades realizan en ellos. Además, en el diseño de las zonas verdes se utilizan con frecuencia patrones subjetivos, muy influenciados por valores estéticos y por modas de una determinada época, que pueden llegar a provocar un desfase entre los utilizadores últimos y los equipamientos proyectados (Puyuelo et al., 2005). Frente a esta situación se contrapone la voluntad de algunos sociólogos, que proponen construir una nueva gobernanza urbana, en la que la toma de decisiones sea flexible, enriqueciendo así la democracia representativa con nuevos procedimientos con los que consultar a los habitantes, a los usuarios y a todos aquellos que tuvieran algo que decir sobre los espacios públicos (Ascher, 2000).

El objetivo de este artículo es identificar qué es lo que está pasando actualmente en el verde urbano, tratando de obtener información inédita sobre los visitantes habituales. Estos espacios surgen como respuesta a necesidades sociales, por lo que será necesario identificarlas para poder realizar un correcto diseño y una adecuada gestión que satisfaga a los usuarios. Esta investigación se ha llevado a cabo en las áreas verdes de tres ciudades españolas de distinto tamaño, tratando de confirmar las hipótesis de que los jóvenes son los mayores utilizadores del verde urbano, de que el periodo del año en que se realizan la mayor parte de las visitas coincide con los meses de más calor y de que la distribución horaria de las mismas se concentran durante la tarde. Se ha tratado de comprobar la influencia del tiempo libre en las visitas y se ha prestado especial atención a las actividades realizadas, identificando a los colectivos que acuden a los espacios verdes para relajarse o para pasear y a los que acuden para cuidar de los niños pequeños.

Para poder desarrollar la investigación aquí propuesta ha sido necesario definir qué es una ciudad y qué se entiende por verde urbano. Las urbes seleccionadas están situadas en la Comunidad Autónoma de Castilla y León, en el noroeste de España, y según el INE, Zamora contaba con 65.998 habitantes en 2010, mientras que Salamanca tenía 154.462 y Valladolid 315.522. Las tres comparten el hecho de ser capitales provinciales y de albergar la mayor parte de los órganos de gobierno provincial, y Valladolid es además sede de las principales instituciones de Castilla y León.

\section{Metodología}

\subsection{Cómo se ha seleccionado el verde urbano}

Es necesario determinar qué es un espacio verde ya que no existe una definición comúnmente aceptada. En este artículo se ha empleado el término de verde urbano y para su definición se ha delimitado la ciudad o lo urbano, seleccionando posteriormente una serie de espacios con vegetación localizados en su interior que pudieran ser visitados por los ciudadanos. A la hora de fijar las fronteras del fenómeno urbano tampoco existe consenso, por lo que se ha optado por utilizar unos límites que fueran operativos al trabajar con entidades de distinto tamaño demográfico y territorial. Para ello se ha utilizado una técnica con la que delimitar el núcleo central de las aglomeraciones urbanas ya que, pese a las nuevas formas de vida, en el interior de las mismas todavía puede distinguirse el lugar central donde se concentran la mayor parte de las residencias y de los puestos de trabajo (Roca, 2003). Para ello se ha reducido la ciudad al municipio vertebrador del área urbana, utilizando la división administrativa actual, empleando también un criterio morfológico que consiste en integrar en una misma aglomeración de espacio construido a las parcelas que presenten discontinuidades espaciales inferiores a $200 \mathrm{~m}$ de suelo no urbano (NEL·Lo y MuÑoz, 2004).

Una vez delimitado el polígono urbano en cada una de las ciudades escogidas, se utilizó una técnica 
con la que seleccionar las áreas verdes que pudieran ser visitadas por toda la población y que tuvieran un tamaño suficiente como para desempeñar alguna de las funciones tradicionales de estos espacios como son el ocio, el deporte, el paseo, etc. (CANosa et al., 2003). Los grandes parques teóricamente recibirían más visitas debido a que el tamaño se encuentra en relación con la cantidad de equipamientos que suelen poseer, lo que contribuye a satisfacer una mayor variedad de necesidades humanas (Van Herzele y Wiedemann, 2003). No se incluyeron las zonas verdes de menores dimensiones por no ser capaces de atraer tantos potenciales usuarios como las de gran tamaño, prescindiendo de los parterres de césped, jardineras e incluso vegetación espontánea en tejados y solares aislados, y de aquellas no frecuentadas debido a su especial localización, como ocurre con las rotondas.

Se ha empleado un método que pone en relación la dimensión de la ciudad y el tamaño de los espacios verdes, ya que la normativa urbanística en vigor en Castilla y León, y su Reglamento de 2009, vinculan la creación de espacios verdes al número total de habitantes de una ciudad y al tamaño de las nuevas piezas urbanas. De esta manera se obtendría un tamaño mínimo del verde urbano con el que realizar comparaciones entre ciudades con dimensiones territoriales y demográficas muy diferentes, que además permitiría trabajar con un número de parques y jardines menor del que gestionan las administraciones locales, analizando así con los espacios verdes de mayor tamaño. La técnica empleada para delimitar el verde urbano consiste en utilizar un layout de cualquier programa de SIG, en este caso el ArcView 3.2, para hacer coincidir los límites de una ciudad con los bordes verticales y horizontales de un A-4. De esta manera se genera una imagen con su correspondiente escala, en la que el divisor permite obtener un valor de referencia del tamaño de la ciudad. Utilizando un coeficiente fijo de $0,3 \mathrm{~m}^{2}$, se multiplicó por el divisor de la escala de la imagen de cada ciudad obteniendo así la superficie mínima del verde. Los resultados de aplicar dicha técnica aparecen en la Tabla I y muestran que el tamaño mínimo del verde urbano en Valladolid es de 1,26 ha, en Salamanca de 0,9 ha y de
0,66 ha en Zamora. Dentro de esta categoría se incluyeron aquellos espacios con una superficie igual o mayor a la obtenida mediante la operación anteriormente referida, con la excepción de los Jardines del Hospital Clínico de Salamanca, que fueron incluidos pese a contar con 0,82 ha. Para analizar la posibilidad de uso del verde urbano, únicamente se ha seleccionado el verde urbano que puede ser visitado por la mayor parte de los ciudadanos, que ha sido denominado como verde urbano disponible. En el caso de Salamanca son 26 espacios, en Valladolid 45 y en Zamora 18 (Figura 1).

\subsection{Cómo se ha realizado el estudio del verde urbano desde una perspectiva social}

Para obtener información sobre el uso que los ciudadanos hacen del verde urbano se recurrió a la técnica de la encuesta personal, obteniendo así información inédita sobre lo que ocurre diariamente en los parques. Es importante destacar que este trabajo se centró exclusivamente en los usuarios de los espacios verdes, dejando a un lado a la parte de la población no usuaria de los mismos. Se realizaron encuestas personales siguiendo un modelo utilizado en un estudio del Vondelpark de Ámsterdam (Chiesura, 2004), con la particularidad de que se decidió realizar los cuestionarios a través de una entrevista directa.

En efecto, se realizaron encuestas seleccionando individuos al azar entre los visitantes del verde urbano disponible, sin efectuar discriminación por su edad, sexo, posición social o sus características profesionales. A la hora de determinar el número de encuestas a realizar, se aplicó el ratio empleado por la citada autora que era de aproximadamente una encuesta por cada mil residentes en la ciudad. Dicha metodología se testó en la ciudad de Salamanca, optándose por añadir cien encuestas más al número obtenido, para así dar solidez a la muestra recogida, de tal manera que se fijó un mínimo de 256 encuestas entre todo el verde urbano disponible con que cuenta la ciudad. Ante el buen resultado obtenido, se mantuvo el ratio mínimo de encuestas en las otras dos ciudades analizadas, que fue de 2,56 encuestas por hectárea, fijando una cifra de 644 encuestas para las 251,4 ha de verde disponible con que

Quadro I

Tamaño mínimo del verde urbano utilizando un coeficiente de $0,3 \mathrm{~m}^{2}$

\begin{tabular}{|c|c|c|c|}
\hline & Escala de la imagen de la ciudad & Coeficiente fijo $\left(\mathrm{m}^{2}\right)$ & Superficie mínima del verde urbano $\left(\mathrm{m}^{2}\right)$ \\
\hline Salamanca & $1 / 30.000$ & 0,3 & 9000 \\
\hline Valladolid & $1 / 42.000$ & 0,3 & 12600 \\
\hline Zamora & $1 / 22.000$ & 0,3 & 6600 \\
\hline
\end{tabular}

Fuente: Elaboración propia. 
contaba Valladolid mientras que en Zamora, fueron 184 las distribuidas entre sus 71,7 ha.

\section{Presentación y análisis de los resultados}

\subsection{Distribución por edad y sexo}

La cifra final de encuestados fue de 1.104 personas, superando el mínimo previsto en la metodología, aunque la distribución en los espacios verdes no concuerda estrictamente con lo planificado, ya que en determinadas ocasiones no fue posible encontrar usuarios suficientes. Las encuestas se distribuyeron de la siguiente manera: 271 en los 26 espacios verdes de Salamanca, 647 en los 44 de Valladolid y 186 en los 18 de Zamora. En el Quadro II se muestra la distribución por grupos de edad y por sexo de la población encuestada, observándose que un $52,4 \%$ son hombres y un $47,6 \%$ mujeres. Esta diferencia de género en el uso de los espacios verdes es especialmente llamativa entre las personas de más de sesenta y cinco años, ya que el número de hombres casi duplica al de mujeres. La explicación de este fenómeno puede encontrarse en los valores tradicionales en los que fue socializado este colectivo, entre ellos existiría una fuerte diferencia de roles en función del sexo, siendo las mujeres de esas edades las que asumen la mayor parte de las labores domésticas (muga, 1980), mientras que los hombres viejos utilizarían parte de su tiempo en realizar visitas a los espacios verdes. Esta diferencia podría incrementarse debido a que el número de mujeres que perciben algunos parques como poco seguros es mayor que el de hombres, lo que redundaría en un menor uso de los mismos (SEGovia y NeIRA, 2005).

Los mayores de sesenta y cinco años representan una tercera parte de la población encuestada, siendo los viejos el grupo más numerosos de todos, mientras que los hombres de entre treinta y cuarenta y cinco años componen el colectivo de usuarios que menos peso tiene entre los encuestados. Para validar estos datos se ha comparado en cada una de las tres ciudades el porcentaje de población entrevistada con el de pobla-

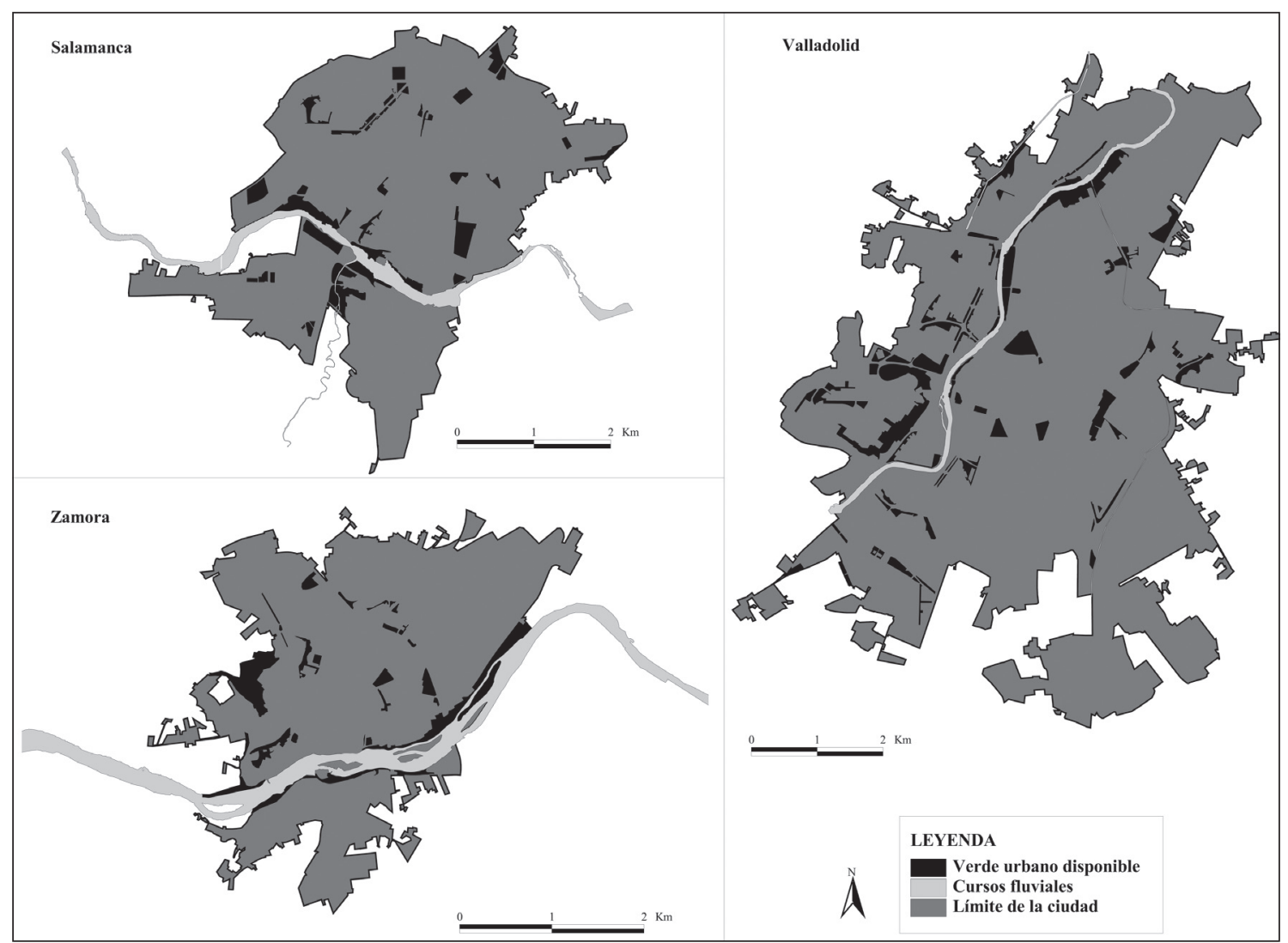

Figura 1

Verde urbano disponible de las ciudades de Salamanca, Zamora y Valladolid.

Fuente: www.Itacyl.es 
Quadro II

Distribución del total de encuestados por edad, sexo y ocupación

\begin{tabular}{|c|c|c|c|c|c|c|c|}
\hline Edad & Ocupación & Hombre & $\%$ Hombre & Mujer & $\%$ Mujer & Total & \% Total \\
\hline \multirow[t]{3}{*}{$18-30$} & Estudia & 98 & 8,9 & 96 & 8,7 & 194 & 17,6 \\
\hline & Trabaja & 32 & 2,9 & 30 & 2,7 & 62 & 5,6 \\
\hline & No empleo & 25 & 2,3 & 9 & 0,8 & 34 & 3,1 \\
\hline \multicolumn{2}{|l|}{ Total $18-30$} & 155 & 14,0 & 135 & 12,2 & 290 & 26,3 \\
\hline \multirow[t]{3}{*}{$30-45$} & Estudia & 1 & 0,1 & 2 & 0,2 & 3 & 0,3 \\
\hline & Trabaja & 65 & 5,9 & 75 & 6,8 & 140 & 12,7 \\
\hline & No empleo & 19 & 1,7 & 55 & 5,0 & 74 & 6,7 \\
\hline \multicolumn{2}{|l|}{ Total $30-45$} & 85 & 7,7 & 132 & 12,0 & 217 & 19,7 \\
\hline \multirow[t]{4}{*}{$45-65$} & Estudia & 0 & 0,0 & 1 & 0,1 & 1 & 0,1 \\
\hline & Trabaja & 69 & 6,3 & 69 & 6,3 & 138 & 12,5 \\
\hline & No empleo & 18 & 1,6 & 53 & 4,8 & 71 & 6,4 \\
\hline & Jubilado & 41 & 3,7 & 27 & 2,4 & 68 & 6,2 \\
\hline \multicolumn{2}{|l|}{ Total 45-65 } & 128 & 11,6 & 150 & 13,6 & 278 & 25,2 \\
\hline \multirow[t]{2}{*}{$>65$} & Trabaja & 4 & 0,4 & 1 & 0,1 & 5 & 0,5 \\
\hline & Jubilado & 207 & 18,8 & 107 & 9,7 & 314 & 28,4 \\
\hline Total $>65$ & & 211 & 19,1 & 108 & 9,8 & 319 & 28,9 \\
\hline Total & & 579 & 52,4 & 525 & 47,6 & 1104 & 100,0 \\
\hline
\end{tabular}

Fuente: Elaboración propia.

ción censada, atendiendo al sexo y a la edad, como se hizo en un estudio sobre la ciudad de Bragança (FonseCA et al., 2010). Para obtener la población real de cada grupo de edad, se sumaron los grupos quinquenales que componen cada uno de ellos, excluyendo a los menores de edad. En este trabajo únicamente se ha trabajado con mayores de dieciocho años ya que es el límite de la mayoría de edad en España. A la hora de comparar los datos obtenidos en las encuestas y los obtenidos del padrón municipal, se produjo un desfase significativo debido a que el INE posee un grupo de edad de entre quince y diecinueve años y otro de entre veinte y veinticuatro. Se optó por excluir el primero de ellos, de tal manera, que en la comparación entre los encues- tados y los censados no se incluyeron los residentes con dieciocho y con diecinueve años, alterándose así la comparación realizada en este grupo de edad. El total de encuestados en esta investigación representaba el $0,21 \%$ de la población con más de veinte años censada en Salamanca en 2010, un 0,25\% en el caso de Valladolid y de un 0,34\% en Zamora.

En la Figura 2 aparecen representadas las diferencia porcentuales entre encuestados y censados, apareciendo de izquierda a derecha, primero los grupos de edad de los hombres y a continuación los de las mujeres. Los valores porcentuales han sido representados en distintas tonalidades y el color negro se ha reservado para el valor medio de las tres ciudades analizadas. Si

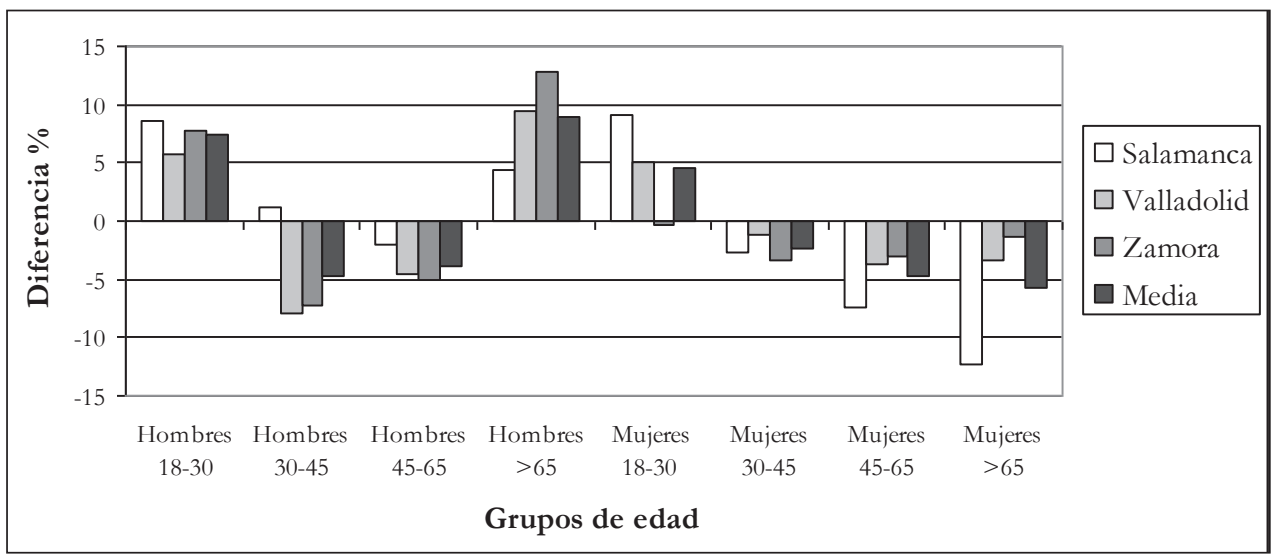

Figura 2

Diferencias entre usuarios encuestados y población censada por grupos de edad y sexo.

Fuente: INE. 
se observa detenidamente el comportamiento de cada uno de los grupos de encuestados, puede identificarse un patrón común que se repite en las tres ciudades, lo que termina por dar validez a la metodología utilizada al seleccionar la muestra poblacional.

La diferencia positiva registrada en el colectivo de jóvenes, tanto de hombres como de mujeres, está influenciada por la exclusión entre los censados de aquellos que tuvieran entre dieciocho y veinte años. Sin embargo, se observa que en este grupo de edad, la media de las tres ciudades estudiadas es un $4,6 \%$ superior a la población censada en el caso de las mujeres y un $7,3 \%$ en el caso de los hombres, lo que refleja que este colectivo es uno de los que más presente se encuentra en los espacios verdes. Por su parte, la población adulta encuestada es inferior a la censada en todos los casos, mostrando cierta homogeneidad entre hombres y mujeres. El dato más llamativo es el enorme contraste existente entre los mayores de sesenta y cinco años, ya que en el promedio de las tres ciudades, la población masculina encuestada superó a la censada en un $8,9 \%$, mientras que la femenina fue claramente inferior. Esto permite afirmar que el elevado porcentaje de hombres viejos encuestados no responde únicamente al significativo envejecimiento de la población en alguna de estas ciudades, sino que muestra una voluntad inequívoca de este grupo de población por utilizar el verde urbano, mientras que entre las mujeres de dicha edad se observa un comportamiento opuesto.

\subsection{Frecuencia y actividades realizadas en los espacios verdes}

El análisis de la frecuencia con que se realizan las visitas a los espacios verdes se llevó a cabo empleando tres preguntas con las que se trató de suplir la enorme labor que habría que realizar para controlar el acceso al verde urbano de las ciudades estudiadas. Con los datos obtenidos se puede afirmar que los usuarios visitan las áreas verdes con mucha frecuencia, puesto que un 67,3\% afirmó realizar cinco o más visitas a la semana, mientras que solamente un 5,1\% aseguró acudir menos de tres veces al mes. La información referente a la ocupación de cada encuestado ha permitido identificar la influencia del tiempo libre en las visitas de cada grupo de individuos, observándose que los jubilados son los que acuden con mayor frecuencia: un $84,6 \%$ de los hombres de esta edad dijo acudir cinco o más veces por semana, mientras que entre las mujeres de esta edad se alcanzó el $75 \%$. De esta manera se comprueba que aunque las señoras jubiladas representen una pequeña parte de la muestra obtenida, las que visitan el verde urbano lo hacen con mucha frecuencia. Otro de los colectivos con bastante tiempo libre es el de las personas desempleadas y tanto las mujeres como los hombres en esta situación acuden con elevada periodicidad a las zonas verdes. Los estudiantes por su parte, acuden con muy poca frecuencia a los espacios verdes, siendo común esta situación para ambos sexos, lo que seguramente esté condicionado por los horarios académicos. Combinando estos datos con los anteriormente mencionados, se llega a la conclusión de que los jubilados suponen un porcentaje de usuarios muy elevado que visita casi todos los días los espacios verdes, mientras que los estudiantes, que representan tan sólo un 17,9\% del total de los encuestados (los jóvenes son el $26,3 \%$ ) visitan los parques con poca frecuencia, acudiendo mayoritariamente una o dos veces por semana.

Las visitas están fuertemente influenciadas por el tiempo, tanto en su sentido cronológico como en su

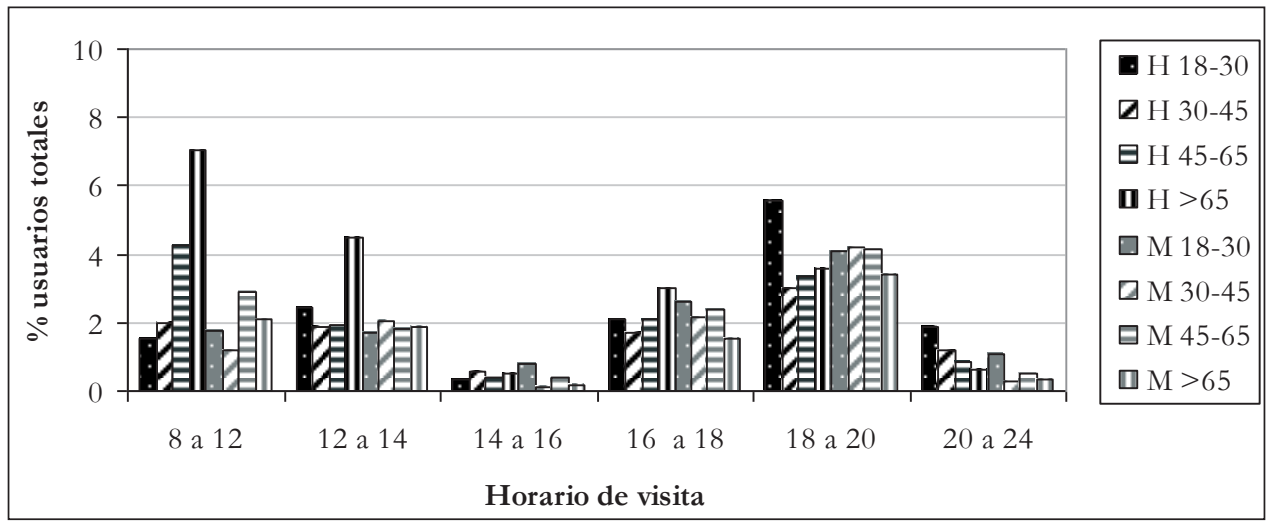

Figura 3

Distribución horaria del total de las visitas a los espacios verdes en función del sexo y de la edad.

Fuente: Elaboración propia. 
sentido climático (CEDEÑo, 2003), como se pudo comprobar en la ciudad de Bragança donde se demostró una relación significativa entre las estaciones cálidas del año y las visitas a las áreas verdes (FonseCA et al., 2010). En las ciudades castellano-leonesas se ha corroborado dicha influencia, puesto que el $92 \%$ de los encuestados dijo acudir en verano y un $82 \%$ en primavera, mientras que en otoño y en invierno la cifra de visitas se redujo a la mitad. De una manera indirecta, esta distribución contribuye a confirmar la hipótesis de que los espacios verdes se utilizan cuando se dispone de tiempo libre. Se registró una mayor concurrencia en el periodo de vacaciones escolares, lo que posiblemente afectará a un $17,9 \%$ de los encuestados que dijeron ser estudiantes, y al $15,5 \%$ que como se analizará más adelante, dijo acudir a los espacios verdes para estar con niños.

La distribución horaria de las visitas muestra una concentración de usuarios durante las horas que trascurren entre la salida y la puesta del sol, siendo muy reducido el colectivo de personas que acude a partir de las ocho de la tarde (Figura 3). La representación horaria, que retomando el símil anterior sería el tiempo en sentido cronológico, muestra que hay dos periodos del día en el que se concentran la mayor parte de las visitas. El primero comprende las primeras horas de la mañana, desde las ocho hasta las doce, en las que los usuarios protagonistas son los hombres de más de sesenta y cinco años que, como se indicó anteriormente, tienen tiempo libre y pueden acudir a los espacios verdes en este horario, estando acompañados de manera menos intensa por hombres adultos-viejos.

El segundo concentra una mayor cantidad de usuarios y tiene una extensión únicamente de dos horas, desde las seis a las ocho de la tarde, durante las cuales acude a los espacios verdes un tercio de los usuarios, especialmente hombres jóvenes y mujeres de entre dieciocho y sesenta y cinco años. Esta última franja horaria sería la utilizada por buena parte de los estudiantes y de las personas que tienen que cuidar de niños, debido a la influencia de las obligaciones académicas, lo que evidencia que el tiempo libre es un condicionante de las visitas al verde urbano. Por otra parte, la población anciana masculina al disponer de mucho tiempo libre presenta una mayor variedad de horarios, pudiendo incluso repetir a lo largo del día. Una vez que se hace de noche, el número de usuarios se reduce de manera sensible debido a que estos espacios comienzan a ser percibidos como poco seguros por una parte importante de la población (CEDEÑo, 2003). Esta autora señala que existe un temor instalado en la socialización femenina, que se transforma en miedo a visitar determinados espacios durante la noche, lo que explicaría que en este estudio sean mayoritariamente hombres los usuarios del verde urbano en este periodo. Es remarcable el hecho de que entre las dos y las cuatro, que coincide con la hora de la comida y de reposo de gran parte de la población, apenas se registren visitas a los espacios verdes. Además, como la mayor parte de los usuarios dijo visitarlos en las estaciones cálidas, hay que pensar que se estarían evitando las horas de más calor, especialmente durante el verano.

La distribución de las visitas está condicionada por las actividades que realizan los distintos usuarios. Estas últimas aparecen representadas en la Figura 4, donde puede observarse, atendiendo a los grandes grupos de edad, que el colectivo de jóvenes acude a las zonas verdes para pasear o realizar actividades deportivas y para relajarse, siendo las primeras preferidas por los

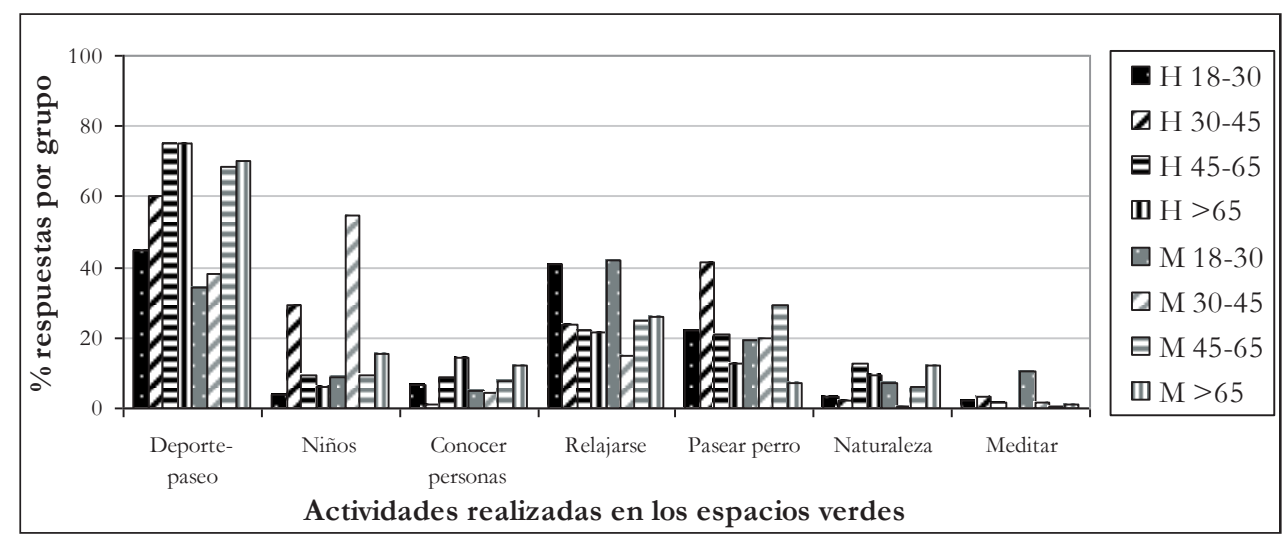

Figura 4

Porcentaje de actividades realizadas en el verde urbano por cada grupo en función del sexo y de la edad.

Fuente: Elaboración propia. 
hombres y la segunda por las mujeres. Por otra parte, el $54,4 \%$ de las mujeres de entre treinta y cuarenta y cinco años acuden a estos espacios para acompañar a sus hijos, siendo este porcentaje mucho más reducido en el caso de los hombres de esta edad. Esto contribuye a explicar el hecho de que este colectivo de mujeres acuda en horario de tarde, ya que lo harán condicionadas por los horarios escolares de sus hijos.

El grupo de adultos-viejos dedica más de dos tercios de sus visitas a la actividad física y menos de un tercio a relajarse o a pasear al perro. Los hombres de entre treinta y cuarenta y cinco años son quienes más desarrollan esta última actividad, mientras que apenas es practicada por los ancianos debido a los cambios asociados al envejecimiento, tales como la menor movilidad articular o la reducción de la fuerza muscular (Puyuelo et al., 2005). Este proceso condiciona las actividades realizadas por las personas mayores, que en su mayor parte pasea o realiza actividades deportivas, y en menor medida a la relajación y a conocer a otras personas, siendo el colectivo que más desarrolla esta tarea socializadora. Las mujeres ancianas representan el tercer grupo poblacional que más acude a las áreas verdes para cuidar niños, dedicando a ello el $15,7 \%$ de las visitas. Esto refleja la importancia de los mayores en el modelo de familia actual, ya que asumen una parte del cuidado de los nietos al existir una implicación generalizada de la pareja en el ámbito laboral (Puyuelo et al., 2005). Otras actividades realizadas en los espacios verdes como escapar de la ciudad, meditar o estar en contacto con la naturaleza son muy poco representativas.

\section{Discusión}

Esta investigación ha permitido identificar una serie de aspectos relevantes del uso que los ciudadanos hacen del verde urbano de Salamanca, Valladolid y Zamora. Se ha comprobado que la mayor parte de los usuarios de estos espacios son hombres, lo que concuerda con los datos publicados sobre la ciudad de Logroño (Muga, 1980), pero destaca especialmente el colectivo de viejos que representa en la muestra un porcentaje mucho más elevado que entre la población censada.

La población encuestada acude con mucha frecuencia a los espacios verdes, ya que más de dos tercios de los encuestados aseguró visitarlos cinco o más veces por semana, al igual que en el estudio realizado en la ciudad portuguesa de Amadora (SANTANA et al., 2010), en el que se afirma que el $83,8 \%$ de los usuarios frecuenta regularmente los áreas verdes. Estas autoras demostra- ron la importancia en la salud de la población de los espacios verdes situados en las proximidades del lugar de residencia, ya que proporcionan un ambiente físico que incentiva la práctica deportiva (SANTANA et al., 2007), confirmando además una fuerte correlación entre proximidad, calidad ambiental y utilización (SANTANA et al., 2011).

Las estaciones cálidas concentran la mayor parte de las visitas y la distribución horaria muestra unas características propias de países mediterráneos, ya que la mayor parte de los asistentes acude en horario de tarde y a primera hora de la mañana, existiendo un vaciado de los parques durante la hora de la comida. Estos datos contrastan con los publicados en un estudio sobre los parques de Viena (Austria), donde la mayor parte de las visitas se concentran entre las doce del mediodía y las cinco de la tarde (ARnBerger, 2006), lo que evidencia una diferenciación posiblemente influenciada por los ritmos u horarios de vida de un país centroeuropeo y de otro mediterráneo, que estarían mediatizados por el clima, por las horas de sol y por los distintos horarios laborales.

Los espacios verdes son lugares de convivencia donde pueden identificarse algunos de los comportamientos más característicos de una sociedad, diferenciando los roles de cada grupo de edad y sexo. De esta manera se observa que las mujeres son quienes se encargan mayoritariamente de las labores familiares: las adultas-jóvenes son quienes acuden con mayor frecuencia acompañadas de sus hijos, mientras que las viejas acuden poco a los espacios verdes ya que tendrían a su cargo la mayor parte de las labores domésticas, dejando así mayor libertada a sus cónyuges para acudir a los espacios verdes. Esta investigación ha demostrado el importante papel de los mayores en el modelo actual de familia, puesto que el $15,7 \%$ de las visitas de las mujeres de este colectivo están dirigidas a cuidar de los más pequeños. Además, los espacios verdes se muestran como lugar de socialización y constituyen uno de los puntos de reunión y estancia de las personas mayores, al igual que se demostró en la ciudad de Castellón (Puyuelo et al., 2005). Conviene también destacar el papel que tienen los espacios verdes para la práctica deportiva en las ciudades donde se llevó a cabo la investigación, marcando una clara diferencia con otros países europeos en los que se prefiere acudir a otros recintos cerrados como gimnasios o pabellones para hacer deporte.

Parece evidente que el tiempo libre es otro de los condicionantes de las visitas a los espacios verdes, puesto que se observó claramente la influencia directa o indirecta de los horarios escolares entre algunos de los grupos de edad y sexo, mientras que los jubilados, 
que disponen de mucho tiempo libre, se identificaron como los mayores usuarios del verde urbano.

\section{Conclusión}

Este trabajo ha permitido obtener información detallada sobre el uso que los ciudadanos hacen de los espacios verdes urbanos empleando una sencilla metodología de trabajo. La técnica empleada para seleccionar el verde urbano de tres ciudades diferentes ha demostrado ser operacional, permitiendo realizar un estudio de las zonas verdes desde un punto de vista social. Los resultados obtenidos concuerdan con diversos estudios llevados a cabo en otras ciudades de la Península Ibérica, lo que ha permitido identificar un comportamiento común de los usuarios del verde urbano.

Este tipo de estudios pueden llegar a ser muy útiles en la fase previa al diseño de los espacios verdes, ya que habitualmente se desconoce quienes son los usuarios últimos de estos equipamientos. Será necesario desarrollar un nuevo urbanismo para evitar una situación como la actual, en la que los ciudadanos no son tenidos en cuenta y no tienen ninguna capacidad de influir en las decisiones que atañen a los espacios que visitan habitualmente (AsCHER, 2000).

\section{Bibliografía}

Arnberger, Arne (2006) - "Recreation use of urban forest: an inter-area comparison". Urban Forestry \& Urban Greening, $n^{\circ} 4$, Amsterdam, pp. 135-144.

AsCHER, François (2010) - Novos princípios do Urbanismo. Livros Horizonte. Lisboa.

Bauman, Zygmunt (2007) - Tiempos líquidos. Tusquets Editores, Barcelona.

Baycan-Levent, Tüzin; Vreeker, Ron e NiJkamp, Peter (2004) Multidimensional Evaluation of Urban Green Spaces: A Comparative Study on European Cities. Serie Research Memoranda / Vrije Universiteit Amsterdam.

Canosa, Elia; Sáez, Ester; Sanabria, Cristina e Zavala, Ignacio (2003) - "Metodología para el estudio de los parques urbanos: la Comunidad de Madrid". Geofocus Revista Internacional de Ciencia y Tecnología de la Información Geográfica, $n^{\circ} 3$, pp. 160-185.

Cedeño, Martha C. (2003) - "Usos y prácticas sociales en un parque público. El caso del parque Metropolitano Les Planes de L'Hospitalet de Llobregat- Barcelona". Zainak. Cuadernos de Antropología-Etnografía, $n^{\circ} 23$, San Sebastián, pp. 545-566.
Chiesura, Anna (2004) - "The role of urban parks for the sustainable city". Landscape and urban planning, $n^{\circ} 68$, pp. 129-138.

FonseCA, Fernando; Gonçalves, Artur e Rodrigues, Orlando (2010) "Comportamentos e percepções sobre os espaços verdes na cidade de Bragança". Finisterra: Revista portuguesa de geografia, Lisboa, vol. 15, n 89, pp. 119-139.

Muga, Montserrat (1980) - "La utilización de los espacios verdes en la ciudad de Logroño". Cuadernos de investigación geográfica, $n^{\circ}$ 6, Logroño, pp. 71-93.

Nel-Lo, Oriol e Muñoz, Francesc (2004) - "El proceso de urbanización". In: Romero, Joan (Ed.) - Geografía humana: procesos, riesgos e incertidumbres en un mundo globalizado. Ariel. Barcelona, pp. 255-332.

PuYuelo, Marina; Gual, Jaume e Galbis, Miguel (2005) - Espacios abiertos urbanos y personas mayores: una experiencia llevada a cabo en distintos parques de la ciudad de Castellón sobre el diseño y su interacción con la población anciana. Universitat Jaume I, Castelló de la Plana.

RocA, Josep (2003) - "La delimitación de la ciudad: ¿una cuestión imposible?". Ciudad y territorio: Estudios territoriales, $n^{\circ} 135$, Madrid, pp. 17-36.

SanesI, Giovanni e Chiarello, Francesco (2006) - "Residents and urban green spaces: The case of Bari”. Urban Forestry Urban Greenning, $n^{\circ}$ 4(3-4), Amsterdam, pp. 125-134.

Santana, Paula; Santos, Rita; Costa, Cláudia e Loureiro, Adriana (2011) - "Urban green spaces and their impact in the health of a population". Rivista Internazionale di Cultura Urbanistica, nº 6, Nápoles, pp. 57-69.

Santana, Paula; Costa, Claudia; Santos, Rita e Loureiro, Adriana (2010) - "O papel dos Espaços Verdes Urbanos no bemestar e saúde das populações". Revista de Estudos Demográficos, $n^{\circ}$ 48, Lisboa, pp. 6-33.

Santana, Paula; Noguelra, Helena; Santos, Rita e Costa, Claudia (2007) - "Avaliação da Qualidade Ambiental dos Espaços Verdes Urbanos no Bem-estar e na Saúde" In: Santana, Paula (Ed.) - A Cidade e a Saúde. Almedina, Coimbra, pp. 219-237.

Segovia, Olga e Neira, Hernán (2005) - "Espacios públicos urbanos: una contribución a la identidad y confianza social y privada". Revista INVI, Vol. $20, \mathrm{n}^{\circ} 55$, Santiago de Chile, pp. 126-182.

Van Herzele, Ann e Wiedemann, Torsten (2003) - "A monitoring tool for the provision of accessible and attractive urban green spaces". Landscape and urban planning, $\mathrm{n}^{\circ} 63$, pp. 109-126.

\section{Recursos electrónicos}

INE (2010) - Padrón municipal. http://www.ine.es 
ITACYL (2010) - Ortofotografías de Castilla y León. http:// www.itacyl.es
UNFPA (2007) - Estado de la población mundial 2007. http:// www.unfpa.org/swp/2007/ 